\title{
Transitioning from video-assisted thoracic surgical lobectomy to robotics for lung cancer: Are there outcomes advantages?
}

\author{
Benjamin E. Lee, MD, ${ }^{\mathrm{a}, \mathrm{b}}$ Robert J. Korst, MD, ${ }^{\mathrm{a}, \mathrm{b}}$ Elaine Kletsman, PA, ${ }^{\mathrm{a}, \mathrm{b}}$ and John R. Rutledge, MAS
}

Objectives: To determine if there are advantages to transitioning to robotics by a surgeon who is already proficient in performing video-assisted thoracic surgical (VATS) lobectomy.

\begin{abstract}
Methods: A single surgeon proficient in VATS lobectomy initiated a robotic lobectomy program, and a retrospective review was conducted of his patients undergoing minimally invasive lobectomy (robotics or VATS) for lung cancer between 2011 and 2012. Data collected included patient/tumor characteristics, morbidity, mortality, operative times, and length of hospital stay.

Results: Over a 24-month period, a total of 69 patients underwent minimally invasive lobectomy (35 robotic, 34 VATS). Patients in each group were similar in age and clinical stage. Robotic upper lobectomy operative times were longer than VATS (172 vs 134 minutes; $P=.001$ ), with no significant difference in lower lobectomies noted ( 140 vs 123 minutes; $P=.1$ ). Median length of stay was 3 days in both groups, and the median number of lymph nodes harvested was 18 (robotic) versus 16 (VATS; $P=.42$ ). Morbidity and mortality for robotic versus VATS were $11 \%$ versus $18 \%(P=.46)$ and $0 \%$ versus $3 \%(P=.49)$, respectively.

Conclusions: There does not seem to be a significant advantage for an established VATS lobectomy surgeon to transition to robotics based on clinical outcomes. The learning curve for robotic upper lobectomies seems to be more significant than that for lower lobectomies. (J Thorac Cardiovasc Surg 2014;147:724-9)
\end{abstract}

Video-assisted thoracic surgical (VATS) lobectomy was first performed in 1992 and has since been proved to be oncologically equivalent and perhaps even superior to traditional lobectomy by thoracotomy. ${ }^{1-3}$ In addition, patients undergoing VATS lobectomy have been shown to have fewer complications, decreased pain, shorter lengths of stay, improved recovery, and better tolerance of adjuvant chemotherapy. ${ }^{4-6}$ However, despite these advantages VATS lobectomy has not been widely used. Recent studies suggest that less than $50 \%$ of lobectomies performed in the United States are performed using VATS. ${ }^{7}$

Robotic lobectomy has recently come to the forefront as a new platform for minimally invasive thoracic surgery. Initial results of robotic lobectomy have shown the same benefits achieved with VATS compared with open thoracotomy are maintained..$^{8-10}$ Moreover, proponents of robotic surgery claim additional benefits of improved ergonomics, three-dimensional optics, and wristed instrument motions, which may allow for a more thorough lymph node dissection. ${ }^{11-13}$ On the other hand, opponents of

From Daniel and Gloria Blumenthal Cancer Center, ${ }^{a}$ Paramus, NJ; and Division of Thoracic Surgery, ${ }^{b}$ Department of Surgery, The Valley Hospital/Valley Health System, Ridgewood, NJ.

Disclosures: Authors have nothing to disclose with regard to commercial support.

Read at the 39th Annual Meeting of The Western Thoracic Surgical Association, Coeur d'Alene, Idaho, June 26-29, 2013

Received for publication June 25, 2013; revisions received Aug 7, 2013; accepted for publication Oct 6, 2013; available ahead of print Nov 18, 2013.

Address for reprints: Benjamin E. Lee, MD, 1 Valley Health Plaza, Paramus, NJ 07652 (E-mail: leebe@valleyhealth.com).

$0022-5223 / \$ 36.00$

Copyright $($ C 2014 by The American Association for Thoracic Surgery

http://dx.doi.org/10.1016/j.jtcvs.2013.10.002 robotic surgery have cited increased costs and longer procedure times. ${ }^{14}$

Despite a growing body of literature regarding robotic lobectomy, there is a paucity of information on whether or not there are advantages to transitioning to robotics by surgeons who are already proficient in performing VATS lobectomy. As a result, we chose to evaluate this transition in the context of our own thoracic surgery program to determine if any advantages exist.

\section{PATIENTS AND METHODS}

\section{Study Design and Patient Cohort}

This study presents a retrospective review of a prospectively collected and maintained surgical database of patients with lung cancers. The Institutional Review Board waived the need for informed consent. From January 2011 to December 2012, all patients who underwent a thoracoscopic lobectomy performed by a single surgeon (B.E.L.) by either conventional VATS (VL) or robotics (RL) for the treatment of biopsy proven or suspected lung cancer were included. In addition, all operations (VL and RL) were performed with the same physician's assistant (E.K.) at the bedside. Patients were excluded if the initial surgical plan for resection was to use a thoracotomy. The determination of the minimally invasive surgical approach was not random in this study. Patients from January 2011 to February 2012 all underwent VL. Our program initiated its RL program in March 2012 and unless scheduling conflicts did not allow for access to robotics, all patients underwent lobectomy by RL from March 2012 until the end of this study. Patients who underwent treatment with neoadjuvant chemotherapy and/or radiation therapy were included.

\section{Surgeon Background}

B.E.L. is a fellowship trained thoracic surgeon competent in VATS lobectomy with more than 200 previous cases. Previous robotics experience included using robotic surgery for thymectomy and mediastinal tumors. No formal training in RL was obtained other than observation of a few cases. 


\section{Abbreviations and Acronyms \\ VATS $=$ video-assisted thoracic surgery \\ VL = VATS lobectomy \\ $\mathrm{RL} \quad=$ robotic lobectomy}

\section{Technique of VATS and RL}

All procedures were performed in the lateral decubitus position with hilar dissection and individual ligation of hilar structures. VATS lobectomy used 2 incisions: a 4-cm anterior utility incision placed in the fourth or fifth intercostal space and 1 additional $12-\mathrm{mm}$ camera port incision as previously described. ${ }^{1}$ Rib spreading was not used in any VATS procedure. For RL, a complete portal system with a 4-armed approach was used (DaVinci S, Intuitive Surgical, Sunnyvale, Calif). One additional 12-mm assist port was placed in the anterior costophrenic region. Three reposable instruments were used for the cases: the PK Dissecting Forceps, a double fenestrated grasper, and the Prograsp forceps (Intuitive Surgical, Sunnyvale, Calif). All hilar structures were individually dissected and ligated using automatic stapling devices. Fissures were completed with either sharp dissection or an automatic stapling device. The anterior costophrenic port was enlarged near the end of the procedure to allow for specimen retrieval. Systematic mediastinal and hilar lymph node dissections were performed for all cases. Lymph node stations addressed typically included paratracheal (4R), tracheobronchial angle (10R), subcarinal (7), interlobar (11) and infrahilar (9) for right-sided resections and periaortic (5), subcarinal (7), tracheobronchial angle (10L), interlobar (11), and infrahilar (9) for left-sided resections. Specimens were placed in impervious bags (Endocatch II 15-mm specimen pouch, Covidien, Norwalk, Conn).

\section{Data Collection and Definitions}

Data collected included patient demographics, age, gender, smoking status, pulmonary function test data, tumor characteristics (location, histology, size, and clinical and pathologic stage), lymph node data (number removed and stations dissected), administration of neoadjuvant therapy, performance of invasive mediastinal staging, operative data (operative time, number of stapler loads used), length of hospital stay, and morbidity and mortality.

Operative time was defined as the time from the initial incision until the completion of wound closure. For robotic cases, this included the time needed for docking and undocking. Conversion was defined as the need to use a rib-spreading thoracotomy or the need to switch from robotic to conventional VATS. All perioperative adverse events were recorded and perioperative deaths were defined as any death occurring during the primary hospitalization or within 30 days after surgery. Prolonged air leaks were defined as lasting 7 days or longer, discharge of a patient with a chest tube in situ, and/or the need to perform bedside pleurodesis. Length of stay was defined using postoperative days. Clinical and pathologic staging was performed using the seventh edition of the American Joint Commission on Cancer staging manual.

\section{Statistical Analysis}

Categorical variables were compared using $\chi^{2}$ tests, and continuous variables were compared using nonparametric Wilcoxon-Mann-Whitney tests. IBM-SPSS Statistics software (version 19) was used for all analyses. ${ }^{13}$

\section{RESULTS}

\section{Patient Characteristics}

Sixty-nine patients met the inclusion criteria for this study. The clinical characteristics of the study cohort are presented in Table 1 . There were 35 patients in the RL group and 34 patients in the VL group. During the period when mainly RL was used, 3 patients underwent VL because of lack of access to robotics. There were no significant differences in age, smoking status, pulmonary function, median tumor size, tumor histology, tumor location, or clinical stage between the 2 groups. However, there was a higher percentage of men in the RL group (Table 1). Neoadjuvant chemoradiotherapy was administered to 4 patients in the RL group; none of the patients underwent this treatment in the VL group.

\section{Operative Results}

There were no conversions to conventional VATS in the robotic group. There was 1 conversion to thoracotomy in the RL group due to the tumor crossing the major fissure and an inability to safely dissect the pulmonary artery. Within the RL group, 1 patient had a bilobectomy (right middle lobe + right lower lobe) and another had a combined lobectomy and segmentectomy (right upper lobe + right lower lobe superior segment). One patient who underwent VATS also had a combined lobectomy and segmentectomy (right lower lobe + right upper lobe posterior segment). No patients required blood transfusions. Differences in operative time, stapler loads used, lymph node data, length of hospital stay, morbidity and mortality between the 2 groups of patients are presented in Table 2 . Within the VL group, 5 patients had nodal upstaging (4 from stage 1 to stage 2, 1 from stage 1 to stage 3 ). No patients in the RL group had nodal upstaging.

With regard to morbidity in the RL group, 2 patients had postoperative atrial fibrillation, 1 had a prolonged air leak, and another developed empyema that was treated by tube thoracostomy. The VL group included 3 patients with prolonged air leaks, 1 patient with severe delirium, and 1 patient requiring bedside bronchoscopy for mucous plugging. One patient also presented 3 weeks after discharge with an acute pulmonary embolism that resulted in death.

\section{Effect of Induction Chemoradiotherapy on Operative Time}

In the 4 patients undergoing RL after receiving induction chemoradiotherapy, the median operating time was 245 minutes, compared with 152 minutes in the 31 patients who did not receive induction therapy $(P=.001)$. Two of these 4 RL patients underwent lobectomies; 1 required a bilobectomy and another required a lobectomy/ segmentectomy.

\section{Effect of Tumor Location on Operative Time}

Figure 1 depicts the 3 -case average times for all robotic lobectomies in this study. The 3 -case average times are shown in Figures 2 and 3 for operative times segregated 
TABLE 1. Patient characteristics $(N=69)$

\begin{tabular}{|c|c|c|c|}
\hline & $\begin{array}{c}\text { VATS } \\
(\mathbf{n}=\mathbf{3 4})\end{array}$ & $\begin{array}{l}\text { Robotic } \\
(\mathbf{n}=35)\end{array}$ & $\begin{array}{c}P \\
\text { value }\end{array}$ \\
\hline Age, median years (range) & $77(52-88)$ & $71(52-84)$ & .06 \\
\hline Male sex, no. (\%) & $8(24)$ & $22(63)$ & .001 \\
\hline \multicolumn{4}{|l|}{ Smoking status, no. $(\%)$} \\
\hline Never & $10(29)$ & $6(17)$ & .46 \\
\hline Former & $9(56)$ & $22(63)$ & \\
\hline Current & $5(15)$ & $7(20)$ & \\
\hline $\mathrm{FEV}_{1} \%$ predicted, median (IQR) & $89(72-100)$ & $81(65-91)$ & .17 \\
\hline DLCo $\%$ predicted, median (IQR) & $77(57.5-95)$ & $81(74-90)$ & .27 \\
\hline \multicolumn{4}{|l|}{ Primary tumor location, no. $(\%)$} \\
\hline Right upper lobectomy & $9(27)$ & $15(43)$ & .43 \\
\hline Right middle lobectomy & $2(6)$ & $1(3)$ & \\
\hline Right lower lobectomy & $10(29)$ & $10(29)$ & \\
\hline Left upper lobectomy & $9(27)$ & $4(11)$ & \\
\hline Left lower lobectomy & $4(12)$ & $5(14)$ & \\
\hline \multicolumn{4}{|l|}{ Histology, no. $(\%)$} \\
\hline Adeno & $27(79)$ & $25(71)$ & .72 \\
\hline Squamous & $4(12)$ & $4(11)$ & \\
\hline Small cell & $1(3)$ & $1(3)$ & \\
\hline Carcinoid & $2(6)$ & $5(14)$ & \\
\hline Tumor size, median cm (range) & $2.5(1.1-13)$ & $2.0(1-14)$ & .32 \\
\hline Mediastinal staging, no. $(\%)$ & $17(50)$ & $16(46)$ & .72 \\
\hline Neoadjuvant therapy, no. $(\%)$ & $0(0)$ & $4(11)$ & .11 \\
\hline \multicolumn{4}{|l|}{ Clinical stage, no. $(\%)$} \\
\hline IA & $19(56)$ & $20(57)$ & .73 \\
\hline IB & $9(26)$ & $6(17)$ & \\
\hline IIA & $3(9)$ & $2(6)$ & \\
\hline IIB & $1(3)$ & $3(9)$ & \\
\hline IIIA & $1(3)$ & $3(9)$ & \\
\hline IVB & $1(3)$ & $1(3)$ & \\
\hline
\end{tabular}

VATS, Video-assisted thoracoscopic surgery; $F E V_{l}$, forced expiratory volume in 1 second; $I Q R$, interquartile range; DLCO, diffusing capacity of lung for carbon monoxide.

by tumor location (specifically upper lobe resections and lower lobe resections). Overall, when comparing RL and $\mathrm{VL}$, there was no difference in the median operative time for lower lobectomies (140 vs 123 minutes, $P=.10$ ). However, for upper lobectomies, the operative time for RL was significantly longer than that for VL (172 vs 134 minutes, $P=.001$ ).

\section{DISCUSSION}

The use of robotics represents an alternative approach to VATS as a minimally invasive operative strategy for lobectomy in patients with lung cancer. Although the literature suggests that RL offers similar advantages over traditional thoracotomy as VL, it is unclear if proficient VL surgeons should invest the time and resources necessary to learn the robotic approach, because they are already practicing a minimally invasive technique. The present study was undertaken to determine if clinically meaningful advantages of RL can be demonstrated over VL, in the context of a single surgeon who was already proficient in
TABLE 2. Perioperative outcomes

\begin{tabular}{|c|c|c|c|}
\hline & $\begin{array}{c}\text { VATS } \\
(\mathbf{n}=\mathbf{3 4})\end{array}$ & $\begin{array}{l}\text { Robotic } \\
(\mathbf{n}=\mathbf{3 5})\end{array}$ & $\begin{array}{c}P \\
\text { value }\end{array}$ \\
\hline $\begin{array}{l}\text { Overall operative time, } \\
\text { median minutes (range) }\end{array}$ & $128(71-237)$ & $161(104-272)$ & $<.001$ \\
\hline $\begin{array}{l}\text { Upper lobectomy, } \\
\text { median minutes (range) }\end{array}$ & $134(92-222)$ & $172(118-240)$ & \\
\hline $\begin{array}{l}\text { Lower lobectomy, } \\
\text { median minutes (range) }\end{array}$ & $123(71-237)$ & $140(104-272)$ & \\
\hline Stapler loads, median no. (range) & $9(5-23)$ & $9.5(5-13)$ & .74 \\
\hline $\begin{array}{l}\text { Lymph nodes removed, } \\
\text { median no. (range) }\end{array}$ & $16(2-44)$ & $18(4-77)$ & .42 \\
\hline $\begin{array}{l}\text { Lymph node stations dissected, } \\
\text { median no. (range) }\end{array}$ & $2(1-3)$ & $3(2-4)$ & .14 \\
\hline Length of stay, median days (IQR) & $3(2-4)$ & $3(2-6)$ & .67 \\
\hline Morbidity, no. (\%) & $6(18)$ & $4(11)$ & .46 \\
\hline Mortality, no. ( $\%)$ & $1(3)$ & $0(0)$ & .49 \\
\hline \multicolumn{4}{|l|}{ Pathologic stage, no. ( $\%)$} \\
\hline IA & $16(47)$ & $22(63)$ & .64 \\
\hline IB & $6(18)$ & $5(14)$ & \\
\hline IIA & $6(18)$ & $2(6)$ & \\
\hline IIB & $3(9)$ & $2(6)$ & \\
\hline IIIA & $2(6)$ & $3(8)$ & \\
\hline IVB & $1(3)$ & $1(3)$ & \\
\hline
\end{tabular}

VATS, Video-assisted thoracoscopic surgery; $I Q R$, interquartile range.

the performance of VL. In this regard, the data show that no demonstrable advantages of RL exist over VL across multiple clinical end points in this setting. The learning curve for robotic upper lobectomy seems to be more significant than that for robotic lower lobectomy. These findings have implications for proficient VATS lobectomy surgeons who are considering transitioning to a robotic approach.

\section{Differences Between VATS and Robotics}

This study demonstrates that there are few differences between RL and VL for multiple clinical end points when an established VATS surgeon initiates an RL program. A clear strength of the present study is that it describes the experience of a single surgeon, thereby eliminating any potential effect of surgeon variability on the results. These findings are consistent with those reported by Jang and colleagues, ${ }^{15}$ where the addition of the robot did not change outcomes over a VATS approach alone. One difference between the 2 approaches in the present study, however, was the number of incisions used: 4 or 5 incisions were used for the robotic cases and 2 in the VATS cases. More incisions may potentially translate into more postoperative pain, however this end point was not measured. No effect of the larger number of incisions was able to be measured in terms of morbidity, and therefore the difference in the number of incisions may not be clinically relevant. Operative time was also longer for RL cases compared with VL. However, this difference was mainly attributable 


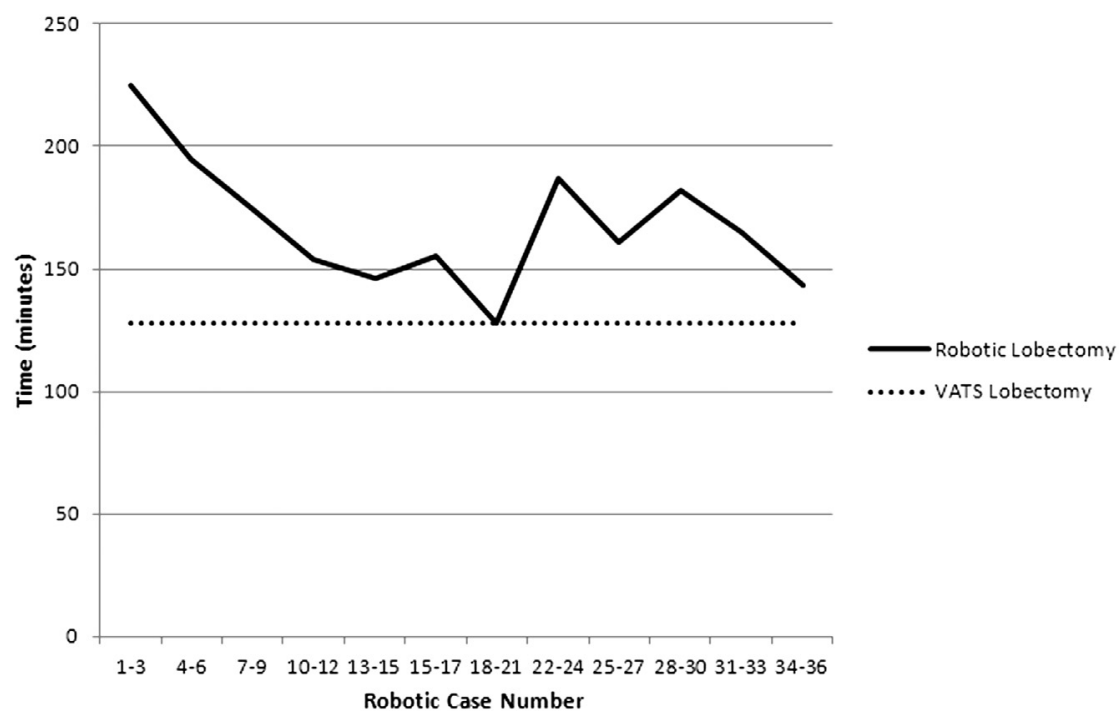

FIGURE 1. Operative time for RL. Three-case average operative case times are shown for consecutive cases and plotted against the overall average for VATS lobectomy. VATS, Video-assisted thoracic surgery.

to the upper lobe robotic cases; no differences were found for lower lobes. Over time, the operative times for upper lobe RL also approached the VL times, suggesting that once the robotic learning curve is completed, minimal difference in operative times may exist.

\section{Learning Curves for Robotic Upper Versus Lower Lobectomy}

From a technical perspective, it is understandable that upper lobe resections are more time consuming as there are typically more individual pulmonary arterial branches that require dissection. Moreover, our technique for lower lobectomy was similar using both VATS and robotic approaches with division of the pulmonary vein followed by the bronchus and finally the pulmonary artery. On the other hand, our VATS upper lobectomies are typically approached from an anterior to posterior dissection (eg, vein, artery, bronchus). We initially performed robotic upper lobectomies in the same manner, but we found visualization and exposure to be difficult at times, which resulted in lengthy case times. As a result of experimenting with different approaches, we have found robotic right upper lobe resections to be more easily approached from the posterior to anterior direction (eg, bronchus, artery, vein) and robotic left upper lobe resections more easily approached through the fissure (lingular artery, vein,

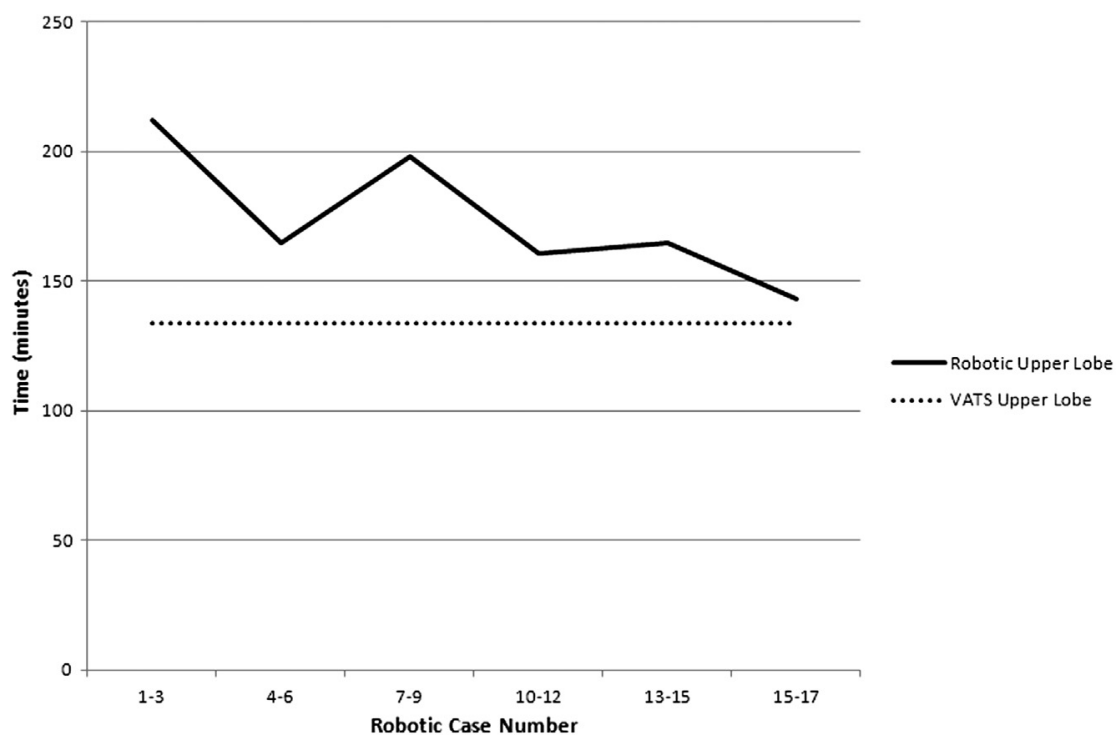

FIGURE 2. Operative time for robotic upper lobectomy. Three-case average operative case times are shown for consecutive cases and compared with the overall average for VATS upper lobectomy. VATS, Video-assisted thoracic surgery. 


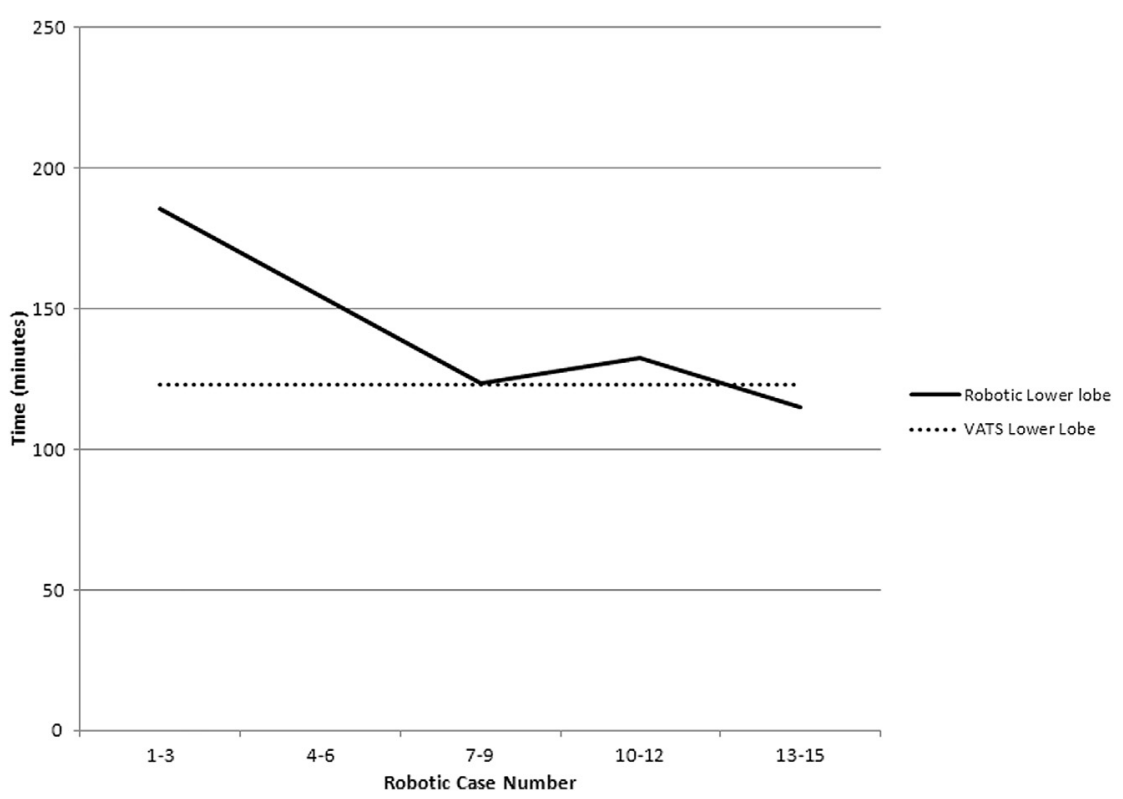

FIGURE 3. Operative time for robotic lower lobectomy. Three-case average operative case times are shown for consecutive cases and compared with the overall average for VATS lower lobectomy. VATS, Video-assisted thoracic surgery.

truncus artery, bronchus, posterior segmental artery). This may have resulted in the more significant learning curve seen with robotic upper lobectomies. Our surgical approach may differ from other centers but it is evident that comparable cases times are achievable for RL.

\section{Costs of RL}

A cost analysis of RL that is applicable to all robotic programs is somewhat problematic because the cost of the robotic platform varies between institutions. The theoretic amortized cost of robotics includes the price of the robotic system divided by the number of cases performed. This varies from one institution to another given that some systems are purchased and others might be donated. Moreover, the number of robotic cases and usage by different surgical disciplines may vary between institutions. Similarly, the cost of a VATS platform also varies between programs. Although it is intuitive that the base cost of robotics is higher than VATS, the ability to assign a universal cost per case of the robot itself is not readily feasible and comparable between institutions.

The present study attempts to address cost from the perspective of tangible costs for RL versus VL. These consist of intraoperative as well as postoperative costs. Intraoperative costs include disposable instrumentation, operative time, and personnel. RL cases in this series only required approximately 30 minutes of additional operative time, and no additional personnel were required over the VL cases. There was also no difference in stapler cartridge usage. From the postoperative standpoint, patients who underwent RL and VL were managed identically, and postoperative length of stay was no different between the procedures. Based on these comparisons, there seemed to be a negligible difference in these costs between RL and VL.

\section{Should an Established VATS Lobectomy Surgeon Learn RL?}

Although the present study demonstrates no clinically significant patient or economic benefits for an established VATS lobectomy surgeon to convert to RL, there may be other reasons not addressed in the present study as to why this transition may be important to consider. First, robotic technology may be advantageous for the performance of other thoracic surgical procedures, and the performance of RL may help keep the surgeon facile at robotic surgery. Second, robotic technology will continue to evolve, and more clearly delineated benefits of robotic surgery may become reality in the future for thoracic surgeons. Third, although not addressed in the present study, the addition of robotics may be beneficial for more advanced cases of lobectomy for lung cancer. Specifically, in cases with difficult nodal dissections, the wristed movements of the robotic instruments combined with the enhanced field of vision associated with robotics may facilitate the minimally invasive resection of more locally advanced cases. However, this remains speculation and needs to be addressed in future studies.

\section{References}

1. Daniels LJ, Balderson SS, Onaitis MW, D’Amico TA. Thoracoscopic lobectomy: a safe and effective strategy for patients with stage I lung cancer. Ann Thorac Surg. 2002;73:860-4.

2. Yamamoto K, Ohsumi A, Kojima F, Imanishi N, Matsuoka K, Ueda M, et al. Long-term survival after video-assisted thoracic surgery lobectomy for primary lung cancer. Ann Thorac Surg. 2010;89:353-9. 
3. Roviaro G, Varoli F, Vergani C, Nucca O, Maciocco M, Grignani F. Long-term survival after videothoracoscopic lobectomy for stage I lung cancer. Chest. 2004; 126:725-32.

4. Paul S, Altorki NK, Sheng S, Lee PC, Harpole DH, Onaitis MW, et al. Thoracoscopic lobectomy is associated with lower morbidity and open lobectomy: a propensity-matched analysis from the STS database. J Thorac Cardiovasc Surg. 2010;132:366-78.

5. Swanson SJ, Meyers BF, Gunnarsson CL, Moore M, Howington JA, Maddaus MA, et al. Video-assisted thoracoscopic lobectomy is less costly and morbid than open lobectomy: a retrospective multiinstitutional database analysis. Ann Thorac Surg. 2012;93:1027-32.

6. Petersen RP, Pham D, Burfeind WR, Hanish SI, Toloza EM, Harpole DH, et al. Thoracoscopic lobectomy facilitates the delivery of chemotherapy after resection for lung cancer. Ann Thorac Surg. 2007;83:1245-9.

7. Boffa DJ, Allen MS, Grab JD, Gaissert HA, Harpole DH, Wright CD. Data from The Society of Thoracic Surgeons General Thoracic Surgery database: the surgical management of primary lung tumors. J Thorac Cardiovasc Surg. 2008; $135: 247-54$.

8. Park BJ, Melfi F, Mussi A, Maisonneuve P, Spaggiari L, Caetano Da Silva RK, et al. Robotic lobectomy for non-small cell lung cancer (NSCLC): long-term oncologic results. J Thorac Cardiovasc Surg. 2012;143:383-9.
9. Louie BE, Farivar AS, Aye RW, Vallieres E. Early experience with robotic lung resection results in similar operative outcomes and morbidity when compared with matched video-assisted thoracoscopic surgery cases. Ann Thorac Surg. 2012;93:1598-604.

10. Cerfolio RJ, Bryant AS, Skylizard L, Minnich DJ. Initial consecutive experience of completely portal robotic pulmonary resection with 4 arms. J Thorac Cardiovasc Surg. 2011;142:740-6.

11. Morgan JA, Ginsburg ME, Sonett JR, Argenziano M. Thoracoscopic lobectomy using robotic technology. Heart Surg Forum. 2003;7:E167-9.

12. Bodner J, Wykypiel H, Wetscher G, Schmid T. First experiences with the da Vinci operating robot in thoracic surgery. Eur J Cardiothorac Surg. 2004;25: 844-51.

13. Park BJ, Flores RM, Rusch VW. Robotic assistance for video-assisted thoracic surgery lobectomy: technique and initial results. J Thorac Cardiovasc Surg. 2006;131:54-9.

14. Flores RM, Alam N. Video-assisted thoracic surgery lobectomy (VATS), open thoracotomy, and the robot for lung cancer. Ann Thorac Surg. 2008;85:S710-5.

15. Jang HJ, Lee HS, Park SY, Zo JI. Comparison of the early robot-assisted lobectomy experience to video-assisted thoracic surgery lobectomy for lung cancer: a single-institution case series matching study. Innovations (Phila) 2011;6:305-10. 